\title{
Oscillation criteria for second-order nonlinear neutral dynamic equations with distributed deviating arguments on time scales
}

\section{Tuncay Candan*}

\section{"Correspondence:}

tcandan@nigde.edu.tr Department of Mathematics, Faculty of Art and Science, Niğde University, Niğde, 51200, Turkey

\section{Abstract}

In this article, we establish some new oscillation criteria and give sufficient conditions to ensure that all solutions of nonlinear neutral dynamic equation of the form

$$
\left(r(t)\left((y(t)+p(t) y(\tau(t)))^{\Delta}\right)^{\gamma}\right)^{\Delta}+\int_{a}^{b} f(t, y(\delta(t, \xi))) \Delta \xi=0
$$

are oscillatory on a time scale $\mathbb{T}$, where $\gamma \geq 1$ is a quotient of odd positive integers.

Keywords: oscillation; dynamic equations; time scales; distributed deviating arguments

\section{Introduction}

The aim of this article is to develop some oscillation theorems for a second-order nonlinear neutral dynamic equation

$$
\left(r(t)\left((y(t)+p(t) y(\tau(t)))^{\Delta}\right)^{\gamma}\right)^{\triangle}+\int_{a}^{b} f(t, y(\delta(t, \xi))) \triangle \xi=0
$$

on a time scale $\mathbb{T}$. Throughout this paper, it is assumed that $\gamma \geq 1$ is a quotient of odd positive integers, $0<a<b, \tau(t): \mathbb{T} \rightarrow \mathbb{T}$, is rd-continuous function such that $\tau(t) \leq t$ and $\tau(t) \rightarrow \infty$ as $t \rightarrow \infty, \delta(t, \xi): \mathbb{T} \times[a, b] \rightarrow \mathbb{T}$ is rd-continuous function such that decreasing with respect to $\xi, \delta(t, \xi) \leq t$ for $\xi \in[a, b], \delta(t, \xi) \rightarrow \infty$ as $t \rightarrow \infty, r(t)>0$ and $0 \leq p(t)<1$ are real valued rd-continuous functions defined on $\mathbb{T}, p(t)$ is increasing and

$\left(\mathrm{H}_{1}\right) \int_{t_{0}}^{\infty}\left(\frac{1}{r(t)}\right) \frac{1}{\gamma} \Delta t=\infty$,

$\left(\mathrm{H}_{2}\right) f: \mathbb{T} \times \mathbb{R} \rightarrow \mathbb{R}$ is a continuous function such that $u f(t, u)>0$ for all $u \neq 0$ and there exists a positive function $q(t)$ defined on $\mathbb{T}$ such that $|f(t, u)| \geq q(t)\left|u^{\gamma}\right|$.

A nontrivial function $y(t)$ is said to be a solution of (1) if $y(t)+p(t) y(\tau(t)) \in C_{r d}^{1}\left[t_{y}, \infty\right]$ and $r(t)\left((y(t)+p(t) y(\tau(t)))^{\Delta}\right)^{\gamma} \in C_{r d}^{1}\left[t_{y}, \infty\right]$ for $t_{y} \geq t_{0}$ and $y(t)$ satisfies equation (1) for $t_{y} \geq t_{0}$. A solution of (1), which is nontrivial for all large $t$, is called oscillatory if it has no last zero. Otherwise, a solution is called nonoscillatory.

C 2013 Candan; licensee Springer. This is an Open Access article distributed under the terms of the Creative Commons Attribution License (http://creativecommons.org/licenses/by/2.0), which permits unrestricted use, distribution, and reproduction in any medium, provided the original work is properly cited. 
We note that if $\mathbb{T}=\mathbb{R}$, we have $\sigma(t)=t, \mu(t)=0, y^{\Delta}(t)=y^{\prime}(t)$ and, therefore, (1) becomes a second-order neutral differential equation with distributed deviating arguments

$$
\left(r(t)\left((y(t)+p(t) y(\tau(t)))^{\prime}\right)^{\gamma}\right)^{\prime}+\int_{a}^{b} f(t, y(\delta(t, \xi))) d \xi=0 .
$$

If $\mathbb{T}=\mathbb{N}$, we have $\sigma(t)=t+1, \mu(t)=1, y^{\Delta}(t)=\Delta y(t)=y(t+1)-y(t)$ and therefore (1) becomes a second-order neutral difference equation with distributed deviating arguments

$$
\triangle\left(r(t)(\triangle(y(t)+p(t) y(\tau(t))))^{\gamma}\right)+\sum_{\xi=a}^{b-1} f(t, y(\delta(t, \xi)))=0
$$

and if $\mathbb{T}=h \mathbb{N}, h>0$, we have $\sigma(t)=t+h, \mu(t)=h, y^{\Delta}(t)=\triangle_{h} y(t)=\frac{y(t+h)-y(t)}{h}$ and, therefore, (1) becomes a second-order neutral difference equation with distributed deviating arguments

$$
\triangle_{h}\left(r(t)\left(\triangle_{h}(y(t)+p(t) y(\tau(t)))\right)^{\gamma}\right)+\sum_{k=\frac{a}{h}}^{\frac{b}{h}-1} f(t, y(\delta(t, k h))) h=0 .
$$

In recent years, there has been important research activity about the oscillatory behavior of second-order neutral differential, difference and dynamic equations. For example, Grace and Lalli [1] considered the following second-order neutral delay equation

$$
\left(a(t)(x(t)+p(t) x(t-\tau))^{\prime}\right)^{\prime}+q(t) f(x(t-\tau))=0, \quad t \geq t_{0}
$$

and Graef et al. [2] considered the nonlinear second-order neutral delay equation

$$
(y(t)+p(t) y(\tau(t)))^{\prime \prime}+q(t) f(y(t-\delta))=0, \quad t \geq t_{0} .
$$

Recently, Agarwal et al. [3] considered second-order nonlinear neutral delay dynamic equation

$$
\left(r(t)\left((y(t)+p(t) y(\tau(t)))^{\triangle}\right)^{\gamma}\right)^{\Delta}+f(t, y(t-\delta))=0 .
$$

Later, Saker [4] considered (2) but he used different technique to prove his results. In [5] and [6], the authors considered the second order neutral functional dynamic equation of the form

$$
\left(r(t)\left((y(t)+p(t) y(\tau(t)))^{\Delta}\right)^{\gamma}\right)^{\Delta}+f(t, y(\delta(t)))=0,
$$

which is more general than (2). For more papers related to oscillation of second-order nonlinear neutral delay dynamic equation on time scales, we refer the reader to [7-10]. For neutral equations with distributed deviating arguments, we refer the reader to the paper by Candan [11]. To the best of our knowledge, [12] is the only paper regarding to 
the distributed deviating arguments on time scales. The books $[13,14]$ gives time scale calculus and some applications.

\section{Main results}

Throughout the paper, we use the following notations for simplicity:

$$
x(t)=y(t)+p(t) y(\tau(t)), \quad x^{[1]}=r\left(x^{\Delta}\right)^{\gamma}, \quad x^{[2]}=\left(x^{[1]}\right)^{\Delta}
$$

and $\theta_{1}(t)=\delta(t, a)$ and $\theta_{2}(t)=\delta(t, b)$.

Theorem 2.1 Assume that $\left(\mathrm{H}_{1}\right)$ and $\left(\mathrm{H}_{2}\right)$ hold. In addition, assume that $r^{\triangle}(t) \geq 0$. Then every solution of $(1)$ oscillates if the inequality

$$
x^{[2]}(t)+A(t) x^{[1]}\left(\theta_{1}(t)\right) \leq 0,
$$

where

$$
A(t)=\frac{(b-a) q(t)\left(1-p\left(\theta_{1}(t)\right)\right)^{\gamma}}{r\left(\theta_{1}(t)\right)}\left(\frac{\theta_{2}(t)}{2}\right)^{\gamma}
$$

has no eventually positive solution.

Proof Let $y(t)$ be a nonoscillatory solution of (1), without loss of generality, we assume that $y(t)>0$ for $t \geq t_{0}$, then $y(\tau(t))>0$ and $y(\delta(t, \xi))>0$ for $t \geq t_{1}>t_{0}$ and $b \geq \xi \geq a$. In the case when $y(t)$ is negative, the proof is similar. In view of $(1),\left(\mathrm{H}_{2}\right)$ and $(3)$

$$
x^{[2]}(t)+\int_{a}^{b} q(t) y^{\gamma}(\delta(t, \xi)) \triangle \xi \leq 0
$$

for all $t \geq t_{1}$, and we see that $x^{[1]}(t)$ is an eventually decreasing function. We claim that $x^{[1]}(t)>0$ eventually. Assume not then there exists a $t_{2} \geq t_{1}$ such that $x^{[1]}\left(t_{2}\right)=c<0$, then we have $x^{[1]}(t) \leq c$ for $t \geq t_{2}$ and it follows that

$$
x^{\Delta}(t) \leq\left(\frac{c}{r(t)}\right)^{1 / \gamma} .
$$

Now integrating (6) from $t_{2}$ to $t$ and using $\left(\mathrm{H}_{1}\right)$, we obtain

$$
x(t) \leq x\left(t_{2}\right)+c^{1 / \gamma} \int_{t_{2}}^{t}\left(\frac{1}{r(s)}\right)^{1 / \gamma} \Delta s \rightarrow-\infty \quad \text { as } t \rightarrow \infty
$$

which contradicts the fact that $x(t)>0$ for all $t \geq t_{0}$. Hence, $x^{[1]}(t)$ is positive. Therefore, one sees that there is a $t_{2} \geq t_{1}$ such that

$$
x(t)>0, \quad x^{\Delta}(t)>0, \quad x^{[1]}(t)>0, \quad x^{[2]}(t)<0, \quad t \geq t_{2} .
$$

For $t \geq t_{3} \geq t_{2}$, this implies that

$$
y(t) \geq x(t)-p(t) x(\tau(t)) \geq(1-p(t)) x(t)
$$


then we conclude that

$$
y^{\gamma}(\delta(t, \xi)) \geq(1-p(\delta(t, \xi)))^{\gamma} x^{\gamma}(\delta(t, \xi)), \quad t \geq t_{4} \geq t_{3}, \xi \in[a, b] .
$$

Multiplying (8) by $q(t)$ and integrating both sides from $a$ to $b$, we have

$$
\int_{a}^{b} q(t) y^{\gamma}(\delta(t, \xi)) \triangle \xi \geq \int_{a}^{b} q(t)(1-p(\delta(t, \xi)))^{\gamma} x^{\gamma}(\delta(t, \xi)) \triangle \xi
$$

Substituting (9) into (5), we obtain

$$
x^{[2]}(t)+\int_{a}^{b} q(t)(1-p(\delta(t, \xi)))^{\gamma} x^{\gamma}(\delta(t, \xi)) \triangle \xi \leq 0 .
$$

On the other hand, we can verify that $x^{\Delta \Delta}(t) \leq 0$ for $t \geq t_{4}$ and, therefore, we obtain

$$
x(t)=x\left(t_{4}\right)+\int_{t_{4}}^{t} x^{\Delta}(s) \triangle s \geq\left(t-t_{4}\right) x^{\Delta}(t) \geq \frac{t}{2} x^{\Delta}(t), \quad t \geq t_{5} \geq 2 t_{4} .
$$

From the last inequality, it can be easily seen that

$$
x(\delta(t, \xi)) \geq\left(\frac{\delta(t, \xi)}{2}\right) x^{\Delta}(\delta(t, \xi)) \geq\left(\frac{\theta_{2}(t)}{2}\right) x^{\Delta}(\delta(t, \xi)), \quad t \geq t_{6} \geq t_{5}, \xi \in[a, b] .
$$

Substituting the last inequality into (10), we have

$$
x^{[2]}(t)+\int_{a}^{b} q(t)(1-p(\delta(t, \xi)))^{\gamma}\left(\frac{\theta_{2}(t)}{2}\right)^{\gamma}\left(x^{\triangle}(\delta(t, \xi))\right)^{\gamma} \triangle \xi \leq 0
$$

and it can be found

$$
x^{[2]}(t)+(b-a) q(t)\left(1-p\left(\theta_{1}(t)\right)\right)^{\gamma}\left(\frac{\theta_{2}(t)}{2}\right)^{\gamma}\left(x^{\Delta}\left(\theta_{1}(t)\right)\right)^{\gamma} \leq 0,
$$

or

$$
x^{[2]}(t)+\frac{(b-a) q(t)\left(1-p\left(\theta_{1}(t)\right)\right)^{\gamma}}{r\left(\theta_{1}(t)\right)}\left(\frac{\theta_{2}(t)}{2}\right)^{\gamma} x^{[1]}\left(\theta_{1}(t)\right) \leq 0,
$$

which is the inequality (4). As a consequence of this, we have a contradiction and therefore every solution of (1) oscillates.

Theorem 2.2 Assume that $\left(\mathrm{H}_{1}\right)$ and $\left(\mathrm{H}_{2}\right)$ hold. In addition, assume that $r^{\Delta}(t) \geq 0, \delta(t, \xi)$ is increasing with respect to $t$ and that the inequality

$$
\limsup _{t \rightarrow \infty} \int_{\theta_{1}(t)}^{t} A(s) \triangle s>1
$$

holds. Then every solution of (1) oscillates. 
Proof Let $y(t)$ be a nonoscillatory solution of (1). We can proceed as in the proof of Theorem 2.1 to get (4). Integrating (4) from $\theta_{1}(t)$ to $t$ for sufficiently large $t$, we have

$$
\begin{aligned}
0 & \geq \int_{\theta_{1}(t)}^{t}\left(x^{[2]}(s)+A(s) x^{[1]}\left(\theta_{1}(s)\right)\right) \triangle s \\
& =x^{[1]}(t)-x^{[1]}\left(\theta_{1}(t)\right)+\int_{\theta_{1}(t)}^{t} A(s) x^{[1]}\left(\theta_{1}(s)\right) \triangle s \\
& \geq x^{[1]}(t)-x^{[1]}\left(\theta_{1}(t)\right)+x^{[1]}\left(\theta_{1}(t)\right) \int_{\theta_{1}(t)}^{t} A(s) \triangle s \\
& =x^{[1]}(t)+x^{[1]}\left(\theta_{1}(t)\right)\left(\int_{\theta_{1}(t)}^{t} A(s) \triangle s-1\right)>0 .
\end{aligned}
$$

By making use of (11), we reach to a contradiction therefore the proof is complete.

Theorem 2.3 Assume that $\left(\mathrm{H}_{1}\right)$ and $\left(\mathrm{H}_{2}\right)$ hold. In addition, assume that $r^{\triangle}(t) \geq 0, \delta(t, \xi)$ is increasing with respect to $t$ and there exists a positive rd-continuous $\Delta$-differentiable function $\alpha(t)$ such that

$$
\limsup _{t \rightarrow \infty} \int_{t_{0}}^{t}\left(\alpha(s) Q(s)-\frac{\left(\left(\alpha^{\Delta}(s)\right)_{+}\right)^{2} r\left(\theta_{2}(s)\right)}{4 \gamma\left(\frac{\theta_{2}(s)}{2}\right)^{\gamma-1} \alpha(s)}\right) \triangle s=\infty,
$$

where $\left(\alpha^{\triangle}(s)\right)_{+}=\max \left\{0, \alpha^{\Delta}(s)\right\}$ and $Q(s)=(b-a) q(s)\left(1-p\left(\theta_{1}(s)\right)\right)^{\gamma}$. Then every solution of (1) is oscillatory on $\left[t_{0}, \infty\right)$.

Proof Suppose to the contrary that $y(t)$ is nonoscillatory solution of (1). We may assume without loss of generality that $y(t)>0$ for $t \geq t_{0}$, then $y(\tau(t))>0$ and $y(\delta(t, \xi))>0$ for $t \geq t_{1}>t_{0}$ and $b \geq \xi \geq a$. Proceeding as in the proof of Theorem 2.1, we obtain (7) and the inequality (10). Using (7) and Pötzsche's chain rule [15, Theorem 1], we obtain

$$
\begin{aligned}
\left(x^{\gamma}(t)\right)^{\Delta} & =\gamma \int_{0}^{1}\left[x(t)+h \mu(t) x^{\Delta}(t)\right]^{\gamma-1} d h x^{\Delta}(t) \\
& \geq \gamma \int_{0}^{1}(x(t))^{\gamma-1} d h x^{\Delta}(t)=\gamma(x(t))^{\gamma-1} x^{\Delta}(t)>0 .
\end{aligned}
$$

From (10) and (13), we obtain

$$
x^{[2]}(t) \leq-(b-a) q(t)\left(1-p\left(\theta_{1}(t)\right)\right)^{\gamma} x^{\gamma}\left(\theta_{2}(t)\right)=-Q(t) x^{\gamma}\left(\theta_{2}(t)\right), \quad t \geq t_{4} .
$$

Define the function

$$
z(t)=\alpha(t) \frac{x^{[1]}(t)}{x^{\gamma}\left(\theta_{2}(t)\right)}, \quad t \geq t_{4}
$$

It is obvious that $z(t)>0$. Taking the derivative of $z(t)$, we see that

$$
\begin{aligned}
z^{\Delta}(t) & =\left(x^{[1]}\right)^{\sigma}(t)\left(\frac{\alpha(t)}{x^{\gamma}\left(\theta_{2}(t)\right)}\right)^{\Delta}+\frac{\alpha(t)}{x^{\gamma}\left(\theta_{2}(t)\right)} x^{[2]}(t) \\
& =\frac{\alpha(t) x^{[2]}(t)}{x^{\gamma}\left(\theta_{2}(t)\right)}+\left(x^{[1]}\right)^{\sigma}(t)\left(\frac{x^{\gamma}\left(\theta_{2}(t)\right) \alpha^{\Delta}(t)-\alpha(t)\left(x^{\gamma}\left(\theta_{2}(t)\right)\right)^{\Delta}}{x^{\gamma}\left(\theta_{2}(t)\right)\left(x^{\sigma}\left(\theta_{2}(t)\right)\right)^{\gamma}}\right) .
\end{aligned}
$$


Now using (14) in (16), we obtain

$$
z^{\triangle}(t) \leq-\alpha(t) Q(t)+\frac{\alpha^{\triangle}(t) z^{\sigma}(t)}{\alpha^{\sigma}(t)}-\frac{\alpha(t)\left(x^{[1]}\right)^{\sigma}(t)\left(x^{\gamma}\left(\theta_{2}(t)\right)\right)^{\triangle}}{x^{\gamma}\left(\theta_{2}(t)\right)\left(x^{\sigma}\left(\theta_{2}(t)\right)\right)^{\gamma}}
$$

On the other hand, as in the proof of Theorem 2.1, it can be shown that for sufficiently large $t \geq t_{5}$

$$
x(t) \geq\left(\frac{t}{2}\right) x^{\Delta}(t), \quad t \geq t_{5} \geq 2 t_{4}
$$

and then

$$
\gamma x^{\gamma-1}(t) \geq \gamma\left(\frac{t}{2}\right)^{\gamma-1}\left(x^{\triangle}(t)\right)^{\gamma-1}
$$

or

$$
\gamma x^{\gamma-1}\left(\theta_{2}(t)\right) \geq \gamma\left(\frac{\theta_{2}(t)}{2}\right)^{\gamma-1}\left(x^{\Delta}\left(\theta_{2}(t)\right)\right)^{\gamma-1}, \quad t \geq t_{6} \geq t_{5} .
$$

Since $x^{[2]}(t)<0$, we have

$$
x^{[1]}(t)>x^{[1]}(\sigma(t))
$$

Multiplying (18) by $x^{\triangle}\left(\theta_{2}(t)\right)$ and using (19), it follows that

$$
\begin{aligned}
\gamma x^{\gamma-1}\left(\theta_{2}(t)\right) x^{\triangle}\left(\theta_{2}(t)\right) & \geq \gamma\left(\frac{\theta_{2}(t)}{2}\right)^{\gamma-1}\left(x^{\triangle}\left(\theta_{2}(t)\right)\right)^{\gamma} \\
& \geq \gamma\left(\frac{\theta_{2}(t)}{2}\right)^{\gamma-1} \frac{r\left(\theta_{2}(\sigma(t))\right)}{r\left(\theta_{2}(t)\right)}\left(x^{\triangle}\left(\theta_{2}(\sigma(t))\right)\right)^{\gamma} \\
& \geq \gamma\left(\frac{\theta_{2}(t)}{2}\right)^{\gamma-1} \frac{\left(x^{[1]}\right)^{\sigma}\left(\theta_{2}(t)\right)}{r\left(\theta_{2}(t)\right)} .
\end{aligned}
$$

From (13), for sufficiently large $t \geq t_{7} \geq t_{6}$, we have

$$
\left(x^{\gamma}\left(\theta_{2}(t)\right)\right)^{\triangle} \geq \gamma x^{\gamma-1}\left(\theta_{2}(t)\right) x^{\triangle}\left(\theta_{2}(t)\right) .
$$

From (20) and (21), it follows that

$$
\left(x^{\gamma}\left(\theta_{2}(t)\right)\right)^{\Delta} \geq \gamma\left(\frac{\theta_{2}(t)}{2}\right)^{\gamma-1} \frac{\left(x^{[1]}\right)^{\sigma}\left(\theta_{2}(t)\right)}{r\left(\theta_{2}(t)\right)} .
$$

Substituting (22) into (17), we obtain

$$
z^{\triangle}(t) \leq-\alpha(t) Q(t)+\frac{\alpha^{\triangle}(t) z^{\sigma}(t)}{\alpha^{\sigma}(t)}-\frac{\gamma\left(\frac{\theta_{2}(t)}{2}\right)^{\gamma-1} \alpha(t)}{\left(\alpha^{\sigma}(t)\right)^{2} r\left(\theta_{2}(t)\right)}\left(z^{\sigma}(t)\right)^{2} .
$$


Using the fact $u-m u^{2} \leq \frac{1}{4 m}, m>0$, we have

$$
\begin{aligned}
z^{\triangle}(t) & \leq-\alpha(t) Q(t)+\frac{\left(\alpha^{\triangle}(t)\right)_{+}}{\alpha^{\sigma}(t)}\left(z^{\sigma}(t)-\frac{\gamma\left(\frac{\theta_{2}(t)}{2}\right)^{\gamma-1} \alpha(t)}{\left(\left(\alpha^{\triangle}(t)\right)_{+}\right) \alpha^{\sigma}(t) r\left(\theta_{2}(t)\right)}\left(z^{\sigma}(t)\right)^{2}\right) \\
& \leq-\left(\alpha(t) Q(t)-\frac{\left(\left(\alpha^{\triangle}(t)\right)_{+}\right)^{2} r\left(\theta_{2}(t)\right)}{4 \gamma\left(\frac{\theta_{2}(t)}{2}\right)^{\gamma-1} \alpha(t)}\right) .
\end{aligned}
$$

Integrating the last inequality from $t_{7}$ to $t$, we obtain

$$
-z\left(t_{7}\right)<z(t)-z\left(t_{7}\right) \leq-\int_{t_{7}}^{t}\left(\alpha(s) Q(s)-\frac{\left(\left(\alpha^{\triangle}(s)\right)_{+}\right)^{2} r\left(\theta_{2}(s)\right)}{4 \gamma\left(\frac{\theta_{2}(s)}{2}\right)^{\gamma-1} \alpha(s)}\right) \triangle s
$$

or

$$
z\left(t_{7}\right)>\int_{t_{7}}^{t}\left(\alpha(s) Q(s)-\frac{\left(\left(\alpha^{\triangle}(s)\right)_{+}\right)^{2} r\left(\theta_{2}(s)\right)}{4 \gamma\left(\frac{\theta_{2}(s)}{2}\right)^{\gamma-1} \alpha(s)}\right) \triangle s
$$

which contradicts (12). Therefore, the proof is complete.

Theorem 2.4 Assume that $\left(\mathrm{H}_{1}\right)$ and $\left(\mathrm{H}_{2}\right)$ hold and $\sigma(t) \neq t$ for each $t \in \mathbb{T}$. Let $\alpha(t), \delta(t, \xi)$, and $Q(s)$ be as defined in Theorem 2.3. If

$$
\limsup _{t \rightarrow \infty} \int_{t_{0}}^{t}\left(\alpha(s) Q(s)-\frac{\left(\left(\alpha^{\triangle}(s)\right)_{+}\right)^{2} r\left(\theta_{2}(s)\right)}{2^{3-\gamma}\left(\mu\left(\theta_{2}(s)\right)\right)^{\gamma-1} \alpha(s)}\right) \triangle s=\infty
$$

then every solution of $(1)$ is oscillatory on $\left[t_{0}, \infty\right)$.

Proof Following the same lines as in the proof of Theorem 2.1, we get (7) and (10). Using the inequality,

$$
x^{\gamma}-y^{\gamma} \geq 2^{1-\gamma}(x-y)^{\gamma}, \quad \gamma \geq 1 \text {, }
$$

we have

$$
\begin{aligned}
\left(x^{\gamma}(t)\right)^{\Delta} & =\frac{x^{\gamma}(\sigma(t))-x^{\gamma}(t)}{\mu(t)} \geq 2^{1-\gamma} \frac{(x(\sigma(t))-x(t))^{\gamma}}{\mu(t)} \\
& =2^{1-\gamma}(\mu(t))^{\gamma-1}\left(\frac{x(\sigma(t))-x(t)}{\mu(t)}\right)^{\gamma}=2^{1-\gamma}(\mu(t))^{\gamma-1}\left(x^{\triangle}(t)\right)^{\gamma} .
\end{aligned}
$$

Now setting $z(t)$ by (15), using (17) and (23) we see that

$$
z^{\triangle}(t) \leq-\alpha(t) Q(t)+\frac{\left(\alpha^{\triangle}(t)\right)_{+} z^{\sigma}(t)}{\alpha^{\sigma}(t)}-\frac{2^{1-\gamma}\left(\mu\left(\theta_{2}(t)\right)\right)^{\gamma-1} \alpha(t)}{\left(\alpha^{\sigma}(t)\right)^{2} r\left(\theta_{2}(t)\right)}\left(z^{\sigma}(t)\right)^{2}
$$

The remaining part of the proof is similar to that of Theorem 2.3, hence it is omitted.

Example 2.5 Consider the following second-order neutral nonlinear dynamic equation

$$
\left(\left(\left(y(t)+\left(\frac{t+a-1}{t+a}\right) y(\tau(t))\right)^{\Delta}\right)^{5 / 3}\right)^{\Delta}+\int_{a}^{b} t^{-1 / 3} y(t-\xi) \Delta \xi=0, \quad t \in \mathbb{T}
$$


where $\gamma=\frac{5}{3}, r(t)=1, p(t)=\left(\frac{t+a-1}{t+a}\right), q(t)=t^{-1 / 3}$. One can verify that the conditions of Theorem 2.3 are satisfied. Note that taking $\alpha(s)=s$, we see that

$$
\begin{aligned}
& \limsup _{t \rightarrow \infty} \int_{t_{0}}^{t}\left(\alpha(s) Q(s)-\frac{\left(\left(\alpha^{\triangle}(s)\right)_{+}\right)^{2} r\left(\theta_{2}(s)\right)}{4 \gamma\left(\frac{\theta_{2}(s)}{2}\right)^{\gamma-1} \alpha(s)}\right) \Delta s \\
& \quad=\limsup _{t \rightarrow \infty} \int_{t_{0}}^{t}\left((b-a) s^{-1}-\frac{1}{\frac{20}{3}\left(\frac{s-b}{2}\right)^{2 / 3} s}\right) \triangle s=\infty .
\end{aligned}
$$

Therefore, (1) is oscillatory.

\section{Competing interests}

The author declares that they have no competing interests.

Received: 26 April 2012 Accepted: 3 April 2013 Published: 18 April 2013

\section{References}

1. Grace, SR, Lalli, BS: Oscillations of nonlinear second order neutral delay differential equations. Rad. Mat. 3, 77-84 (1987)

2. Graef, JR, Grammatikopoulos, MK, Spikes, PW: Asymptotic properties of solutions of nonlinear neutral delay differential equations of the second order. Rad. Mat. 4(1), 133-149 (1988)

3. Agarwal, RP, O'Regan, D, Saker, SH: Oscillation criteria for second-order nonlinear neutral delay dynamic equations. J. Math. Anal. Appl. 300, 203-217 (2004)

4. Saker, SH: Oscillation of second-order nonlinear neutral delay dynamic equations on time scales. J. Comput. Appl. Math. 187, 123-141 (2006)

5. Saker, $\mathrm{SH}$ : Oscillation criteria for a second-order quasilinear neutral functional dynamic equation on time scales. Nonlinear Oscil. 13, 407-428 (2011)

6. Saker, SH, O'Regan, D: New oscillation criteria for second-order neutral functional dynamic equations via the generalized Riccati substitution. Commun. Nonlinear Sci. Numer. Simul. 16(1), 423-434 (2011)

7. Saker, SH: Oscillation of superlinear and sublinear neutral delay dynamic equations. Commun. Appl. Anal. 12(2), 173-187 (2008)

8. Saker, SH, Agarwal, RP, O'Regan, D: Oscillation results for second-order nonlinear neutral delay dynamic equations on time scales. Appl. Anal. 86, 1-17 (2007)

9. Saker, SH, O'Regan, D, Agarwal, RP: Oscillation theorems for second-order nonlinear neutral delay dynamic equations on time scales. Acta Math. Sin. Engl. Ser. 24, 1409-1432 (2008)

10. Saker, SH: Hille and Nehari types oscillation criteria for second-order neutral delay dynamic equations. Dyn. Contin. Discrete Impuls. Syst., Ser. B, Appl. Algorithms 16(3), 349-360 (2009)

11. Candan, T, Dahiya, RS: On the oscillation of certain mixed neutral equations. Appl. Math. Lett. 21(3), $222-226$ (2008)

12. Candan, T: Oscillation of second-order nonlinear neutral dynamic equations on time scales with distributed deviating arguments. Comput. Math. Appl. 62(11), 4118-4125 (2011)

13. Bohner, M, Peterson, A: Dynamic Equations on Time Scales: An Introduction with Applications. Birkhäuser, Boston (2001)

14. Bohner, M, Peterson, A: Advances in Dynamic Equations on Time Scales. Birkhäuser, Boston (2003)

15. Pötzsche, C: Chain rule and invariance principle on measure chains. J. Comput. Appl. Math. 141, $249-254$ (2002)

doi:10.1186/1687-1847-2013-112

Cite this article as: Candan: Oscillation criteria for second-order nonlinear neutral dynamic equations with distributed deviating arguments on time scales. Advances in Difference Equations 2013 2013:112.

\section{Submit your manuscript to a SpringerOpen ${ }^{\ominus}$ journal and benefit from:}

- Convenient online submission

- Rigorous peer review

- Immediate publication on acceptance

Open access: articles freely available online

- High visibility within the field

- Retaining the copyright to your article 